BULL. AUSTRAL. MATH. SOC.

$|6 A 2|$

VOL. 12 (1975), 95-97.

\title{
Sub-prime radical classes determined by zerorings
}

\author{
B.J. Gardner
}

It is shown that the correspondence which associates with each radical class $T$ of abelian groups the (radical) class of prime radical rings with additive groups in $T$ gives a complete classification of those radical classes of rings which are determined (as lower radicals) by zerorings.

In this note we investigate radical subclasses of the Baer lower (= prime) radical class $B . B$ is the lower radical class defined by the class of all zerorings, so we are interested in the extent to which rings in the lower radical class over a class of zerorings are describable in terms of their membership of $B$ and the (additive) structure of the zerorings concerned.

In what follows, $G^{0}$ is the zeroring on an abelian group $G, R^{+}$ the additive group of a ring $R$ and $L(C)$ the lower radical class defined by a class $C$ of rings. (For details concerning radicals, see [3].)

Let $T$ be a radical class of abelian groups, $T^{0}=\left\{G^{0} \mid G \in T\right\}$, $T^{*}=\left\{R \mid R^{+} \in T\right\}$. Then $T^{*}$ is a radical class and thus there are two ways of associating a radical subclass of $B$ with $T$ : one can consider $L\left(T^{0}\right)$ or $B \cap T^{*}$. (For further details, see [4].) It is clear that $L\left(T^{0}\right) \subseteq B \cap T^{*}$ in all cases, so the obvious problem is to determine when we have equality here. Armendariz [1] showed that equality holds when $T$

Received 29 October 1974. 
is subgroup-closed and this was extended by the author [4] to the case where $T$ is merely closed under pure subgroups. In this paper we demonstrate equality for every $T$.

Since for any radical class $R$ the class of zerorings in $R$ has the form $T^{0}$, and since, moreover, a nilpotent ring $A$ belongs to a radical class $R$ if and only if $A^{+0} \in R$ ([5], Theorem 2.5) our result provides a characterization of the lower radical classes defined by classes of nilpotent rings.

We make use of a notion introduced by Sands [7] in his investigation of the interaction between radicals and Morita contexts. A class $C$ of rings is principally left hereditary if $R a \in C$ for all $a \in R \in C$.

All rings considered are associative; the symbol 4 indicates an ideal.

PROPOSITION 1. Let $T$ be a radical class of abelion groups. Then $B \cap T^{*}$ is principally left hereditary.

Proof. If $a \in R \in B \cdot \cap T^{*}$, then $R a^{+}$is a homomorphic image of $R^{+}$ via the correspondence $r \mapsto r a$, so $R a \in T^{*}$. Also $B$ is subring-closed and hence $R a \in B$. //

PROPOSITION 2. Let $R$ be a principally left hereditary radical subclass of $B, M$ the class of nilpotent mings in $R$. Then $R=L(M)$.

Proof. By Theorem 3 of [6], $L(M)$ consists of all rings $A$ such that every non-zero homomorphic image $A^{\prime \prime}$ has a non-zero accessible subring $S$ in $M$; that is, there exists a finite chain

$$
0 \neq S=I_{1} \triangleleft I_{2} \triangleleft \ldots \triangleleft I_{n} \triangleleft A^{\prime \prime} \text {. }
$$

Since $R$ is homomorphically closed, it is enough to show that non-zero rings in $R$ have non-zero accessible subrings in $M$ and clearly only nonnilpotent rings need be considered. Such a ring $R$ does not coincide with its right annihilator $(R: 0)$, so $R /(R: 0)$ is a non-zero ring in $B$ and accordingly has a non-zero nilpotent ideal $I$. Let $\bar{a}$ be a non-zero element of $I$ represented in $R$ by $a$. Then

$$
\operatorname{Ra} /[\operatorname{Ran}(R: 0)] \cong[\operatorname{Ra}+(R: 0)] /(R: 0) \subseteq I,
$$

so $R a /[\operatorname{Ran}(R: 0)]$ is nilpotent, whence it follows that $R a$ is 
nilpotent. Also $R a \neq 0$, and, since $R$ is principally left hereditary, $R a \in M$. Finally, let $J /(R: 0)=I$. Then $J$ is nilpotent and $R a \subseteq J$. By Proposition 8 of [2], $R a$ is an accessible subring of $R$.//

THEOREM. Let $T$ be a radical class of abelian groups. Then $L\left(T^{0}\right)=B \cap T^{*}$.

Proof. A nilpotent ring $A$ belongs to a radical class $R$ if and only if $A^{+0}$ does ([5], Theorem 2.5) and $\left\{G \mid G^{0} \in R\right\}$ is a radical class of abelian groups ([4], Proposition 1.1). Combining these observations with Propositions 1 and 2 , we see that $B \cap T^{*}=L\left(U^{0}\right)$ for some radical class $U$ of abelian groups. But $U^{0} \subseteq B \cap T^{*}$ implies $u \subseteq T$, while $T^{0} \subseteq B \cap T^{*}=L\left(U^{0}\right) \subseteq B \cap U^{*}$ implies $T \subseteq U$. This proves the theorem. //

Note that we have also shown that a radical subclass of $B$ is principally left hereditary if and only if it has the form $B \cap T^{*}$.

\section{References}

[1] E.P. Armendariz, "Hereditary subradicals of the Iower Baer radical", Publ. Math. Debrecen 15 (1968), 91-93

[2] Reinhold Baer, "Meta ideals", Report of a conference on linear algebras, 33-52 (National Research Council Publication 502. National Academy of Sciences, Washington, 1957).

[3] Nathan J. Divinsky, Rings and radicals (University of Toronto Press, Toronto; Allen \& Unwin, London; 1965).

[4] B.J. Gardner, "Radicals of abelian groups and associative rings", Acta Math. Acad. Sci. Hiongar. 24 (1973), 259-268.

[5] B.J. Gardner, "Some sspects of T-nilpotence", Pacific J. Math. (to appear).

[6] Yu-Lee Lee, "On the construction of lower radical properties", Pacific J. Math. 28 (1969), 393-395.

[7] A.D. Sands, "On normal radicals", submitted.

Department of Mathematics, University of Tasmania, Hobart, Tasmania. 